\title{
Management Strategies to Reduce Risk of Postoperative Infections
}

\author{
Anat Galor · Raquel Goldhardt • Sarah R. Wellik • \\ Ninel Z. Gregori · Harry W. Flynn
}

Published online: 18 August 2013

(C) Springer Science + Business Media New York 2013

\begin{abstract}
Postoperative infections, although rare, are still of great concern to the ophthalmologist. The incidence of post-cataract endophthalmitis is low, with a range of 0.28 per 1,000 to 2.99 per 1,000 . In addition to intraoperative considerations such as poor wound construction, vitreous loss, topical anesthesia, and prolonged surgical time, other risk factors include preoperative factors such as a diseased ocular surface and systemic immunosuppression. Potential methods of reducing risk of endophthalmitis after anterior segment surgery are discussed and available literature is summarized.
\end{abstract}

Keywords Endophthalmitis - Cataract surgery - Ocular surface disease $\cdot$ Intracameral antibiotics $\cdot$ Sterilization

\section{Introduction}

Postoperative infections, although rare, are still of great concern to the ophthalmologist. This paper will review the literature, with a focus on more recent studies, and address the incidence and risk factors for postoperative endophthalmitis after cataract and other anterior segment surgery. Based on this, recommendations for pre-, peri-, and

A. Galor - R. Goldhardt - S. R. Wellik · N. Z. Gregori Miami Veterans Administration Medical Center, 1201 NW 16th St, Miami, FL 33125, USA

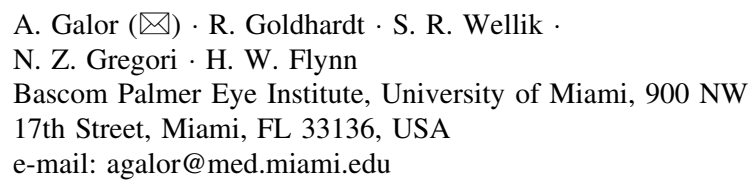

postoperative strategies that may decrease the risk of infection will be reviewed.

\section{Incidence of Postoperative Infections}

Several studies have examined the incidence of endophthalmitis after cataract surgery. Fortunately, the incidence is low, as has been demonstrated by several well-performed retrospective studies throughout the world (Table 1). Endophthalmitis rates ranged from the lowest of 0.28 per 1,000 in a study by Wykoff et al. [15・•] from Miami to the highest of 2.99 per 1,000 cases in a study by Melo [49] from Brazil.

\section{Preoperative Considerations}

\section{The Ocular Surface}

The status of the ocular surface is believed to play a role in post-cataract ocular infections. In a consecutive study of 1,474 conjunctival swabs taken before surgery, $15 \%$ of patients $(n=214)$ had a positive culture [1]. The presence of a local ocular abnormality including chronic use of topical medications, contact lens wear, blepharitis, and/or chronic eyelid or conjunctival inflammation was a risk factor for bacterial recovery. In 282 patients without local abnormalities, $14(5 \%)$ had a positive conjunctival culture compared to $23.5 \%$ (118/503 patients) with a local risk factor [1]. Furthermore, patients with the above risk factors are more likely to harbor antibiotic-resistant conjunctival organisms [2]. While a positive culture does not directly translate into endophthalmitis, these data suggest that it is important to look for and treat moderate to severe 
Table 1 Recent studies reviewing incidence of endophthalmitis after cataract surgery

\begin{tabular}{llll}
\hline Publication & Location & Time period reviewed & Endophthalmitis rate \\
\hline Wykoff et al. [15••] & Bascom Palmer Eye Hospital, Miami, FL, USA & $2002-2009$ & 0.28 per 1,000 (8/28,568) \\
Friling et al. [4••] & Swedish National Cataract Register & $2005-2010$ & 0.29 per 1,000 (135/464,996) \\
Jambulingam et al. [40] & Teriary care hospital, Tamil Nadu, India & $2000-2007$ & 0.53 per 1,000 (70/132,075) \\
Keay et al. [47] & US Medicare beneficiary claims data & $2003-2004$ & 1.22 per 1,000 (4,006/3,280,966) \\
Freeman et al. [48] & Quebec State Control for Health Insurance, Canada & $1996-2005$ & 1.5 per 1,000 (754/490,690) \\
Melo et al. [49] & Universidade Federal de Sao Paulo, Brazil & $2002-2008$ & 2.99 per 1,000 (73/24,590) \\
\hline
\end{tabular}

blepharitis prior to cataract surgery. The treatment approach should mirror disease severity with lid hygiene for mild cases and oral doxycycline/minocycline for more severe disease.

\section{Immunosuppression}

Similar to local ocular abnormalities, the presence of systemic abnormalities including immunosuppression, diabetes, autoimmune conditions, and asthma is a risk factor for culture positivity and for harboring multi-drug-resistant organisms $[1,2]$. In one study, patients with a systemic abnormality had an $11.9 \%(65 / 545)$ frequency of bacterial recovery compared to $5 \%(14 / 282)$ in the control group, $p=0.002$. In this study, two patients developed postoperative endophthalmitis $(0.14 \%)$, one with both local and systemic risk factors and another with a systemic risk factor alone [1]. Furthermore, the use of immunosuppressant agents was found to increase the risk of post-cataract endophthalmitis is a case-control study [3]. A waning immune system may also partially explain the higher rates of endophthalmitis seen in older individuals [4••, 5], although older patients are also more likely to have culturable bacteria on their conjunctivae [1]. While immunosuppression is frequently a non-modifiable risk factor, it has been suggested to place a suture through the corneal wound in patients with a compromised immune system such as those undergoing chemotherapy or who are postbone marrow transplant.

\section{Perioperative Considerations}

\section{Antibiotic Use}

There is wide variation among regions with respect to perioperative antibiotic use. Several studies have shown decreased endophthalmitis rates with the use of intracameral antibiotics (Table 2).

In 2002, a US evidence-based review on bacterial endophthalmitis prophylaxis for cataract surgery reported that the only method of effective prophylaxis was the use of povidone-iodine $5 \%$ in the conjunctival sac just before surgery [6]. However, the presence of anterior chamber contamination after surgery has been noted in eyes even after the application of povidone-iodine $5 \%$ solution prior to surgery. In one study of 100 eyes treated with iodine, 3 had a positive anterior chamber culture (Pseudomonas oryzihabitans in one eye, Klebsiella pneumoniae in one eye, and yeast in one eye). No patient developed endophthalmitis [34••]. Several studies in Europe, including the European Society of Cataract \& Refractive Surgeons (ESCRS) multi-centered study, have demonstrated a reduced incidence of endophthalmitis with the use of intracameral antibiotics at the end of surgery [4••, 7-9]. In a prospective, randomized multi-centered study, 16,603 patients underwent cataract surgery and received one of four treatment regimens (povidone iodine alone or povidone iodine plus an additional therapy, or intracameral cefuroxime or topical levofloxacin, or both). Twenty-nine patients presented with endophthalmitis, of which 20 were culture-positive. The absence of intracameral cefuroxime (1 $\mathrm{mg}$ in $0.1 \mathrm{~mL}$ normal saline) was associated with a $4.92-$ fold increase [5 \% confidence interval (CI), 1.87-12.9] in the risk for total postoperative endophthalmitis [9]. Several other studies in Europe and Asia have replicated these findings (Table 2) [4••, 7, 8]. For example, a study from Sweden reported the risk of endophthalmitis was 13.6 times higher (95\% CI 7.7-24) in those not treated with cefuroxime. Various antibiotics have been studied rates including cefuroxime [4••, 7, 10], cefazolin [8, 11•], moxifloxacin, and vancomycin [12••] all showing the same reduction in endophthalmitis rates. One limitation of all studies except the ESCRS is that they compare cataract surgeries performed at different time periods. Patients who received intracameral antibiotics had their surgeries in the more recent years compared to those who did not receive this therapy. It is possible that other factors, other than antibiotic use, changed in the studied time period and accounts for the decreased endophthalmitis rates seen in the later dates.

Despite these well conducted studies, the widespread use of intracameral antibiotics has not been adopted in the US, mainly due to concerns over the use of compounded 
Table 2 Endophthalmitis rates with and without the use of intracameral antibiotics

\begin{tabular}{|c|c|c|c|c|}
\hline Publication & Country & $\begin{array}{l}\text { Time period } \\
\text { reviewed }\end{array}$ & $\begin{array}{l}\text { Endophthalmitis rate without } \\
\text { intracameral antibiotics }\end{array}$ & $\begin{array}{l}\text { Endophthalmitis rate with } \\
\text { intracameral antibiotics }\end{array}$ \\
\hline $\begin{array}{l}\text { Friling et al. } \\
{[4 \bullet \bullet]}\end{array}$ & Swedish National Cataract Register & $2002-2010$ & 3.9 per $1,000(11 / 2,804)$ & 0.27 per $1,0001(23 / 461,951)$ \\
\hline $\begin{array}{l}\text { Shorstein et al. } \\
{[12 \bullet \bullet]}\end{array}$ & $\begin{array}{l}\text { Kaiser Permanente, Diablo Service } \\
\text { Area, California, USA }\end{array}$ & $2007-2011$ & 4.20 per $1,000(15 / 3,655)$ & 0.32 per $1,000(4 / 12,609)$ \\
\hline $\begin{array}{l}\text { Barrau et al. } \\
\text { [7] }\end{array}$ & Dupuytren Hospital, Limoges, France & $2003-2008$ & 12.4 per $1,000(35 / 2,826)$ & 0.44 per $1,000(1 / 2,289)$ \\
\hline $\begin{array}{l}\text { Romero-Aroca } \\
\text { et al. [8] }\end{array}$ & $\begin{array}{l}\text { Hospital Universitari Sant Joan, } \\
\text { Reus, Spain }\end{array}$ & 1996-2009 & 6.3 per $1,000(76 / 13,305)$ & 0.5 per $1,000(7 / 11,696)$ \\
\hline Tan et al. $\left[11^{\bullet}\right]$ & Tan Tock Seng Hospital, Singapore & 1999-2010 & 0.64 per $1,000(19 / 29,539)$. & 0.1 per $1,000(2 / 20,638)$ \\
\hline $\begin{array}{l}\text { Garcia-Saenz } \\
\text { et al. [10] }\end{array}$ & $\begin{array}{l}\text { University Hospital Fundacion } \\
\text { Alcorcon, Madrid, Spain }\end{array}$ & 1999-2008 & 5.9 per $1,000(39 / 6,595)$ & 0.43 per $1,000(3 / 7,057)$ \\
\hline
\end{tabular}

medications and increasing antimicrobial resistance. No commercially available antibiotic is available for intracameral use, and compounding carries a high risk of infection if strict aseptic guidelines are not followed. The concerns over compounding were recently brought to light by Goldberg et al. [13], who reported 12 cases of Streptococcus mitis/ oralis endophthalmitis caused by contaminated intravitreal bevacizumab prepared by a single compounding pharmacy in South Florida. Centers for Disease Control and Prevention reported a series of six post-cataract surgery endophthalmitis cases due to Gram-negative bacteria associated with contaminated trypan blue dye from a compounding pharmacy. Unopened trypan blue syringes yielded Pseudomonas aeruginosa and Burkholderia cepacia complex on culture [13]. Nine cases of Fusarium solani endophthalmitis were reported in Istanbul, presumably due to contaminated cefuroxime [14•]. This medication was obtained from the same vial and injected intracamerally at end of surgery. All patients developed acute postoperative endophthalmitis [14•].

Moreover, there are concerns over increasing rates of antibiotic resistance in the context of publications reporting low rates of infection without intracameral antibiotics [15••]. Ta et al. [16] reported that less than $70 \%$ of the isolated coagulase-negative Staphylococcus from conjunctiva of patients undergoing anterior segment surgery were sensitive to the penicillin analogues, ceftazidime, erythromycin, and tetracycline. A study from the Bascom Palmer Eye Institute, where no intracameral antibiotics were used, demonstrated one of the lowest endophthalmitis rates published to date, supporting a notion that meticulous preoperative preparation with povidone-iodine is an effective agent for infection prophylaxis [15••]. Given the recent scares of devastating endophthalmitis cases after the use of compounded products and concerns over toxic anterior segment syndrome, US surgeons are hesitant to adopt the routine use of an off-label product which requires compounding. The general consensus among US surgeons is to use povidone-iodine immediately prior to surgery, with varying regimens for topical antibiotics before, during, and after surgery.

\section{Anesthesia}

The use of topical anesthesia has been identified as a potential risk factor for endophthalmitis [17]. In a retrospective study from a single-center academic practice in Spain, the incidence of endophthalmitis was lower in cataract extractions performed under retrobulbar anesthesia (1.3 per 1,000) compared to topical (8.7 per 1,000) (relative risk, 6.72; $95 \%$ CI, 1.63-27.63). One concern using gel for anesthesia prior to invasive ocular procedures is that, if applied prior to povidone-iodine, it may prevent povidone's access to conjunctival flora and potentially increase the risk for endophthalmitis. For this reason, it has been recommended to place povidone-iodine prior to anesthetic gel [18].

\section{Wound Construction}

Sutureless cataract incisions allow for rapid visual rehabilitation after phacoemulsification, and clear corneal incisions have become increasingly popular among ocular surgeons. However, some studies suggest that clear corneal wounds may be associated with an increased risk of postoperative endophthalmitis [19, 20]. Studies have demonstrated that, in the first hours after surgery, self-sealing corneal wounds exhibit a dynamic morphology. In wellpressurized eyes, the wound margins remain well apposed along the length of the incision. However, fluctuation in intraocular pressure (IOP) within a physiologic range $(5-40 \mathrm{mmHg})$ results in movement and gaping of the wound edge, particularly at the internal aspect of the incisions, with posterior migration of the posterior and peripheral wound leaflet [21]. Variables that influenced this 
movement included low IOP and intrinsic wound characteristics [22]. A retrospective cohort study showed that wound leak on the first operative day increased the risk of postoperative endophthalmitis 44-fold [23]. This finding makes sense, given the fact that increased intraocular exposure to one's own normal adnexal and ocular surface flora is one of the main risk factors for endophthalmitis [24].

Earlier work showed that incisions that are square in surface architecture are significantly more resistant to deformation and leakage than those that are rectangular [25]. Based on this finding, surgeons should attempt to create a wound that is the same length as the width of their keratome. Furthermore, the angle of entry influences wound gap with deeper wounds (more perpendicular to the cornea) sealing better with lower IOP and shallower wounds (less perpendicular to cornea) sealing better with higher IOP [21, 22]. While inferior wound location has been postulated as a potential risk factor for endophthalmitis due to its proximity with the tear meniscus [18], several studies have failed to demonstrate an association between wound location and infection [18, 26-28]. Intrastromal wound hydration at the end of the procedure can improve wound sealing; however, the effect is transient and can last as little as $20 \mathrm{~min}$ [29] or up to 1 week [30•].

Interestingly, side-port incisions (SPIs) have also been shown to leak, and care must be taken to ensure good apposition of all surgical wounds. One study examined India ink penetration versus topical fluorescein and noted that India ink penetration occurred in wounds without fluorescein leakage [23]. This, of course, raises the concern that topical fluorescein may not be adequate in assessing the wound integrity.

Preliminary study in cadaveric eyes showed that corneal tunnel incisions constructed with femtosecond laser technology could provide a more stable wound architecture with less postoperative wound gap [31]. Whether this might impact endophthalmitis risk would be worthy of future study. In summary, well-constructed wounds, a wellpressurized eye, and watertight closure at the end of the case (determined by depressing the posterior lip of the incision) are critical in reducing the risk of acute postoperative endophthalmitis.

\section{Phacoemulsification Time}

A recent case-control study evaluated risk factors for postoperative endophthalmitis caused by Pseudomonas aeruginosa and included 8 cases and 24 controls operated on from 2005 to 2009 . Longer phacoemulsification time was found to be significant associated with infection $(p=0.001)[32 \bullet]$.

\section{Retained Cortical Material}

An in vitro study examined the growth of Staphylococcus aureus and epidermidis in aqueous humor that had variable amounts of human lens cortex added. An increased growth of both organisms was found in aqueous with increasing amounts of lens cortex. The authors suggested that eyes with residual lens cortex may be at higher risk of developing endophthalmitis if bacterial contamination occurs during phacoemulsification [33].

\section{Vitreous Presentation}

Posterior capsular rupture and vitreous presentation are well-known risk factors for endophthalmitis [4, 5]. On a related manner, increased operative time has also been identified as a possible risk factor [17]. While improving surgical skills, especially during the training period, decreases the risk of intraocular complications and operative time $[35,36]$, there are no data on which manipulations should be adopted to decrease the risk of endophthalmitis in complicated cases. Empirically, however, it makes sense to suture the corneal wound to increase the integrity of the globe, minimizing potential risk of a wound leak and further vitreous loss.

\section{Lens Choice}

The ESCRS study reported that placement of a silicone intraocular lens (IOL) increased the risk of endophthalmitis 3.13-fold compared to acrylic material (95\% CI 1.47-6.67). Our general practice is to place a one-piece acrylic lens in the capsular bag when allowable. Threepiece acrylic lenses are used for ciliary sulcus placement and poly(methyl methacrylate) (PMMA) lenses are used for lens placement in the anterior chamber. Silicone lenses are typically reserved for select situations such as placement of a piggy-back lens in the sulcus in a patient with significant refractive surprise after surgery. Silicone lenses also present visualization challenges during retinal procedures, such as poor visualization under air and silicone oil adhesion, and therefore we prefer not to routinely implant them.

Proper Sterilization and Handling of Equipment

The need for proper sterilization and handling of equipment has been demonstrated by several endophthalmitis outbreaks due to contaminated material. In India, 11 patients developed acute postoperative Pseudomonas endophthalmitis due to contaminated of hydrophilic intraocular lens solution [37]. Eight cases of acute postoperative 
endophthalmitis (Stenotrophomonas maltophilia and Cellulosimicrobium cellulans) developed in Turkey over a 1-month period, related to contaminated irrigating solution [38]. A contaminated air-conditioning system was the probable cause of an outbreak of 20 Pseudomonas endophthalmitis cases after cataract surgery in Tamil Nadu, India [39]. One case of endophthalmitis in a tertiary care hospital in Tamil Nadu, India, was traced back to a contaminated phaco probe [40].

Based on this, it is important to follow all standard protocols defined by the Association of Perioperative Registered Nurses (AORN) and recommendations by Centers for Disease Control (CDC). These including cleaning and sterilization of instruments, equipment and environmental cleanliness, aseptic technique, discarding single use devices, staff training and competency, and appropriate storage and use of medications and solutions. Ongoing surveillance of the sterilization practices and maintenance of staff competencies are vital to maintenance of robust aseptic practices. A team consisting of surgery, environmental services and infectious control personnel assures a multifaceted approach to achieving standards maintenance. An infectious control committee, working closely with providers and nursing staff, is critical to gathering data on all postoperative infections and investigating potential causes that can be mitigated on the institutional level. For example, the Infection Prevention and Control Practitioners in Miami-Dade County have a communication system to alert one another if an infection is identified that may be related to another facility.

\section{Postoperative Considerations}

Postoperative systemic status may influence the risk of endophthalmitis. A study from Hyderabad, India, found a higher risk of acute endophthalmitis after cataract surgery in patients with lower socioeconomic status [41•]. This finding may be due to limited access to hygienic conditions or concomitant systemic infections. We recently had a patient who developed pneumonia and diarrhea immediately after uncomplicated cataract extraction. He presented with light perception vision and a hypopyon (Fig. 1) 10 days after surgery (Fig. 1). Cultures from the vitrectomy cassette revealed Enterococcus faecalis. While we cannot definitively tie the ocular infection to his systemic status, it is certainly plausible that his eye became contaminated during the course of his illness. General hygiene measures must be stressed to all postoperative patients. Such measures include frequent hand-washing and avoiding touching the eye, swimming, and medication bottle contamination.

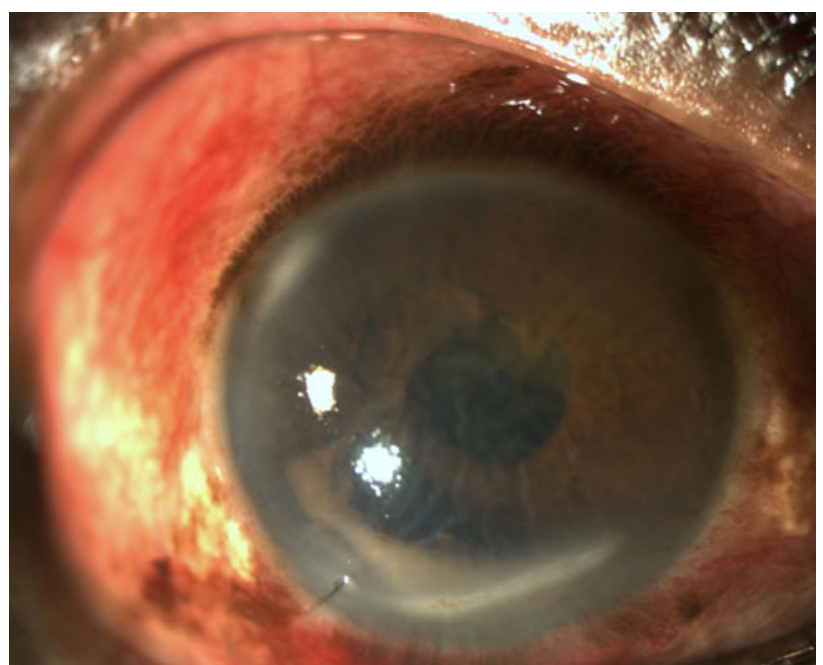

Fig. 1 Slit lamp photograph demonstrating corneal edema and a hypopyon in a patient with decreased vision and pain 10 days after cataract surgery. Systemically, patient became ill with pneumonia and diarrhea after his surgery

\section{Non-cataract Surgery}

There are a number of considerations for infection prevention in non-cataract surgery. Glaucoma surgery in particular presents some challenges for infection prevention. Glaucoma patients are known to have an increased frequency of ocular surface disease (OSD) due to the use of topical intraocular pressure lowering medications. Many of these medications contain Benzalkonium chloride (BAK) which is known to cause surface epithelial defects. However, even non-BAK-containing glaucoma drops are associated with OSD. As noted above, OSD may increase susceptibility to ocular infections in the perioperative period.

Glaucoma surgery, by its very nature of attempting to create a method of fluid egress from the eye, increases the chances of bacterial infiltration into the eye. The reported incidence of post-trabeculectomy endophthalmitis is higher than for cataract surgery. An analysis of the Collaborative Initial Glaucoma Treatment Study (CIGTS) examined 247 patients who had trabeculectomy and at least 5 years follow up. The 5-year rate of blebitis was $3 \%$, and the rate of endophthalmitis was $1.1 \%[42 \bullet \bullet]$. A recent report from the UK demonstrated a $0.17 \%$ incidence of endophthalmitis after trabeculectomy surgery $(n=12)$ over a 13-month period from 2007 to 2008 [43]. The incidence of blebitis without endophthalmitis was $0.1 \%(n=13)$. Another report investigating trabeculectomy-associated endophthalmitis in a Chinese population found a rate of $0.1 \%$ for early onset endophthalmitis and $0.6 \%$ for late onset endophthalmitis at 5 years. In that study, the use of 
Mitomycin C significantly increased the risk of late-onset endophthalmitis $(p=0.0002)$ [44].

The use of antimetabolites in glaucoma surgery has been shown to produce significantly lower intraocular pressures and possibly a higher frequency of hypotony and bleb leak. A recent Cochrane review of 11 trials with 698 participants, however, did not show a higher risk of sightthreatening complications, including endophthalmitis, with the use of these medications because none of the trials were large enough or of sufficient duration to adequately study this potential complication [45]. In our practice, while we feel that the risk of bleb-related infections is higher with antimetabolites, we do routinely use mitomycin $\mathrm{C}$ with the majority of trabeculectomy procedures in order to achieve appropriate postoperative intraocular pressures.

Standard ophthalmic surgical preparations are used for glaucoma surgery similar to cataract surgery. In combined cataract and glaucoma surgery, sutures are routinely placed at the cataract incision site even if the wound is apparently watertight. Target intraocular pressure after glaucoma surgery is often intentionally low (often below 10) which may in turn diminish the integrity of the watertight cataract wound. In the early postoperative period, one must be vigilant to look for bleb leaks which may be a path for microbes into the eye. In the early post-surgical period, these are prophylactically treated with topical antibiotics, but, if leaks persist, they must be actively managed with bandage contact lens and/or aqueous suppressant medications and, if non-healing, surgically repaired.

Placement of an aqueous humor shunt presents an additional concern due to foreign material permanently residing inside the eye. Overall 5-year rates of endophthalmitis have been reported at $1.7 \%$ in a large study by Al-Torbak et al. [46], which reviewed 542 cases of Ahmed glaucoma valve placement between 1994 and 2003. In that study, endophthalmitis was delayed (6 weeks or more after surgery) in eight of nine eyes. The synthetic material of the implants (most commonly silicone) is thought to provide a surface for biofilm formation and may potentiate ocular infections. Aqueous humor shunts are traditionally covered with a patch graft to prevent erosion of the plastic material through the conjunctiva. A variety of materials are used for this purpose, including processed donor pericardium, processed donor sclera, eye bank sclera, and eye bank cornea. In our practice, eye bank material is most often used, and these tissues are soaked in betadine and then dehydrated in glycerine which is thought to kill any potential infectious microorganisms. In addition, the donor subject is serologically tested for several strains of hepatitis, human immunodeficiency virus, and blood cultures are tested for bacterial sepsis. There have been no reported cases of infection due to contaminated patch graft material from our eye bank tissue in the last 15 years.
Another potential source of infection in glaucoma patients is the use of office-based interventions after surgery including injection of $5 \mathrm{FU}$, removing releasable trabeculectomy sutures, bleb needling procedures, placement of bleb compression sutures, reformation of the anterior chamber with fluid or viscoelastic agents, and placement of a bandage contact lens. As such, all these procedures should be performed with standard anti-infective techniques as would be done for other ophthalmic procedures (i.e. injection of intravitreal agents). In recent years, there has been a push toward "minimally invasive glaucoma surgery" which includes: endocyclophotocoagulation, viscocanalostomy, canaloplasty, deep sclerectomy, trabectome, and iStent (Glaukos). While the goal of these procedures has been, in part, to avoid some of the complications of traditional glaucoma surgery, there are not yet sufficient data to properly evaluate rates of infection.

In summary, in patients undergoing glaucoma surgery or combined cataract and glaucoma surgery, it is important to pre-operatively optimize the ocular surface, use standard operative preparation, place a 10-0 nylon suture even if the wound is apparently water-tight, and vigilantly observe for any bleb or wound leaks after surgery.

\section{Conclusions}

Endophthalmitis can be a devastating complication after ocular surgery. While not entirely preventable, considerable knowledge has been gained about ways to decrease the risk of infection. The following measures should be considered:

- treating ocular surface disease prior to surgery

- preoperative povidone-iodine antisepsis

- meticulous surgical technique

- proper equipment sterilization techniques

- intracameral antibiotics at the end of surgery

- patient education on proper hygiene in the immediate postoperative period

Disclosure Anat Galor has been a consultant to B\&L. Raquel Goldhardt declares that she has no conflict of interest. Sarah R. Wellik declares that she has no conflict of interest. Ninel Z. Gregori declares no conflict of interest. Harry W. Flynn has been a consultant to Santen Inc. and Vindico.

Human and Animal Rights and Informed Consent This article does not contain any studies with human or animal subjects performed by any of the authors.

Funding Supported by a VA career development award (Dr. Galor), NIH Center Core Grant P30EY014801, Research to Prevent 
Blindness Unrestricted Grant, Department of Defense (DODGrant\#W81XWH-09-1-0675) and NIEHS (ES014004-01A2)

\section{References}

Papers of particular interest, published recently, have beenhighlighted as:

- Of importance

- Of major importance

1. Mino De Kaspar H, Ta CN, Froehlich SJ, et al. Prospective study of risk factors for conjunctival bacterial contamination in patients undergoing intraocular surgery. Eur J Ophthalmol. 2009;19: 717-22.

2. Mino de Kaspar H, Shriver EM, Nguyen EV, et al. Risk factors for antibiotic-resistant conjunctival bacterial flora in patients undergoing intraocular surgery. Graefes Arch Clin Exp Ophthalmol. 2003;241:730-3.

3. Montan PG, Koranyi G, Setterquist HE, et al. Endophthalmitis after cataract surgery: risk factors relating to technique and events of the operation and patient history: a retrospective case-control study. Ophthalmology. 1998;105:2171-7.

4. •- Friling E, Lundstrom M, Stenevi U, Montan P. Six-year incidence of endophthalmitis after cataract surgery: Swedish national study. J Cataract Refract Surg. 2013;39:15-21. A comprehensive study of all cataract surgeries performed in Sweden showing a decrease in the endophthalmitis rate after the universal adoption of cefuroxime intracameral antibiotic administration after cataract surgery.

5. Hatch WV, Cernat G, Wong D, et al. Risk factors for acute endophthalmitis after cataract surgery: a population-based study. Ophthalmology. 2009;116:425-30.

6. Ciulla TA, Starr MB, Masket S. Bacterial endophthalmitis prophylaxis for cataract surgery: an evidence-based update. Ophthalmology. 2002;109:13-24.

7. Barreau G, Mounier M, Marin B, et al. Intracameral cefuroxime injection at the end of cataract surgery to reduce the incidence of endophthalmitis: French study. J Cataract Refract Surg. 2012;38:1370-5.

8. Romero-Aroca P, Mendez-Marin I, Salvat-Serra M, et al. Results at seven years after the use of intracameral cefazolin as an endophthalmitis prophylaxis in cataract surgery. BMC Ophthalmol. 2012;12:2

9. Endophthalmitis Study Group, European Society of Cataract \& Refractive Surgeons. Prophylaxis of postoperative endophthalmitis following cataract surgery results of the ESCRS multicenter study and identification of risk factors. J Cataract Refract Surg. 2007;33:978-88.

10. Garcia-Saenz MC, Arias-Puente A, Rodriguez-Caravaca G, Banuelos JB. Effectiveness of intracameral cefuroxime in preventing endophthalmitis after cataract surgery ten-year comparative study. J Cataract Refract Surg. 2010;36:203-7.

11. - Tan CS, Wong HK, Yang FP. Epidemiology of postoperative endophthalmitis in an Asian population: 11-year incidence and effect of intracameral antibiotic agents. J Cataract Refract Surg. 2012;38:425-30. First Asian study to demonstrate a reduction in endophthalmitis rates after the universal adoption of intracameral cefazolin administration after cataract surgery in Tan Tock Seng Hospital, Singapore.

12. •• Shorstein NH, Winthrop KL, Herrinton LJ. Decreased postoperative endophthalmitis rate after institution of intracameral antibiotics in a northern California eye department. J Cataract Refract Surg. 2013;39:8-14. The first US study to demonstrate a reduction in endophthalmitis rates after the universal adoption of intracameral antibiotic administration after cataract surgery in the Kaiser Permanente patients in northern California.

13. Goldberg RA, Flynn HW, Jr., Isom RF, et al. An outbreak of Streptococcus endophthalmitis after intravitreal injection of bevacizumab. Am J Ophthalmol. 2012;153(2):204-8 e1.

14. - Gungel H, Eren MH, Pinarci EY, et al. An outbreak of Fusarium solani endophthalmitis after cataract surgery in an eye training and research hospital in Istanbul. Mycoses. 2011;54:e767-74. A study demonstrating the danger of using compounded medications as 9 patients developed devastating endophthalmitis likely in the setting of contaminated cefuroxime.

15. •• Wykoff CC, Parrott MB, Flynn HW, Jr., et al. Nosocomial acute-onset postoperative endophthalmitis at a university teaching hospital (2002-2009). Am J Ophthalmol. 2010;150:392-8 e2. A retrospective study reporting the lowest rate of endophthalmitis in the literature, achieved without the use of intracameral antibiotics.

16. Ta CN, Chang RT, Singh K, et al. Antibiotic resistance patterns of ocular bacterial flora: a prospective study of patients undergoing anterior segment surgery. Ophthalmology. 2003;110: 1946-51.

17. Garcia-Arumi J, Fonollosa A, Sararols L, et al. Topical anesthesia: possible risk factor for endophthalmitis after cataract extraction. J Cataract Refract Surg. 2007;33:989-92.

18. Miller JJ, Scott IU, Flynn HW Jr, et al. Acute-onset endophthalmitis after cataract surgery (2000-2004): incidence, clinical settings, and visual acuity outcomes after treatment. Am J Ophthalmol. 2005;139:983-7.

19. Nagaki Y, Hayasaka S, Kadoi C, et al. Bacterial endophthalmitis after small-incision cataract surgery. Effect of incision placement and intraocular lens type. J Cataract Refract Surg. 2003;29:20-6.

20. Colleaux KM, Hamilton WK. Effect of prophylactic antibiotics and incision type on the incidence of endophthalmitis after cataract surgery. Can J Ophthalmol. 2000;35:373-8.

21. McDonnell PJ, Taban M, Sarayba M, et al. Dynamic morphology of clear corneal cataract incisions. Ophthalmology. 2003;110: 2342-8.

22. Taban M, Rao B, Reznik J, et al. Dynamic morphology of sutureless cataract wounds-effect of incision angle and location. Surv Ophthalmol. 2004;49 Suppl 2:S62-72.

23. Wallin T, Parker J, Jin Y, et al. Cohort study of 27 cases of endophthalmitis at a single institution. J Cataract Refract Surg. 2005;31:735-41.

24. Packer M, Chang DF, Dewey SH, et al. Prevention, diagnosis, and management of acute postoperative bacterial endophthalmitis. J Cataract Refract Surg. 2011;37:1699-714.

25. Ernest PH, Kiessling LA, Lavery KT. Relative strength of cataract incisions in cadaver eyes. J Cataract Refract Surg. 1991;17(Suppl):668-71.

26. Mollan SP, Gao A, Lockwood A, et al. Postcataract endophthalmitis: incidence and microbial isolates in a United Kingdom region from 1996 through 2004. J Cataract Refract Surg. 2007;33: $265-8$.

27. Ng JQ, Morlet N, Bulsara MK, Semmens JB. Reducing the risk for endophthalmitis after cataract surgery: population-based nested case-control study: endophthalmitis population study of Western Australia sixth report. J Cataract Refract Surg. 2007;33: 269-80.

28. Lundstrom M, Wejde G, Stenevi U, et al. Endophthalmitis after cataract surgery: a nationwide prospective study evaluating incidence in relation to incision type and location. Ophthalmology. 2007;114:866-70. 
29. Francis IC, Roufas A, Figueira EC, et al. Endophthalmitis following cataract surgery: the sucking corneal wound. J Cataract Refract Surg. 2009;35:1643-5.

30. • Fukuda S, Kawana K, Yasuno Y, Oshika T. Wound architecture of clear corneal incision with or without stromal hydration observed with 3-dimensional optical coherence tomography. Am J Ophthalmol. 2011;151:413-9 e1. A study demonstrating the transient nature of the effect of stromal wound hydration in clear corneal incisions.

31. Masket S, Sarayba M, Ignacio T, Fram N. Femtosecond laserassisted cataract incisions: architectural stability and reproducibility. J Cataract Refract Surg. 2010;36:1048-9.

32. - Luz RA, Padoveze MC, Falabella P, et al. Risk factors for postoperative endophthalmitis caused by Pseudomonas aeruginosa: Possible role of environment. Am J Infect Control. 2013. A study suggesting that the operating room environment may play a role in the risk of post-cataract endophthalmitis.

33. Lou B, Lin X, Luo L, et al. Residual lens cortex material: potential risk factor for endophthalmitis after phacoemulsification cataract surgery. J Cataract Refract Surg. 2013;39:250-7.

34. • Simaroj P, Kompreyarat S, Santanirand P, Lekhanont K. Anterior chamber contamination during phacoemulsification after povidone-iodine application. $\mathrm{J}$ Med Assoc Thai. 2012;95:689-92. A study demonstrating that anterior chamber bacterial contamination can occur even after the application of povidone-iodine.

35. Taravella MJ, Davidson R, Erlanger M, et al. Characterizing the learning curve in phacoemulsification. J Cataract Refract Surg. 2011;37:1069-75.

36. Lee JS, Hou CH, Yang ML, et al. A different approach to assess resident phacoemulsification learning curve: analysis of both completion and complication rates. Eye (Lond). 2009;23:683-7.

37. Ramappa M, Majji AB, Murthy SI, et al. An outbreak of acute post-cataract surgery Pseudomonas sp. endophthalmitis caused by contaminated hydrophilic intraocular lens solution. Ophthalmology. 2012;119:564-70.

38. Akcakaya AA, Sargin F, Erbil HH, et al. A cluster of acute-onset postoperative endophthalmitis over a 1-month period: investigation of an outbreak caused by uncommon species. Br J Ophthalmol. 2011;95:481-4.
39. Pinna A, Usai D, Sechi LA, et al. An outbreak of post-cataract surgery endophthalmitis caused by Pseudomonas aeruginosa. Ophthalmology. 2009;116:2321-6 e1-4.

40. Jambulingam M, Parameswaran SK, Lysa S, et al. A study on the incidence, microbiological analysis and investigations on the source of infection of postoperative infectious endophthalmitis in a tertiary care ophthalmic hospital: an 8-year study. Indian J Ophthalmol. 2010;58:297-302.

41. - Das T, Hussain A, Naduvilath T, et al. Case control analyses of acute endophthalmitis after cataract surgery in South India associated with technique, patient care, and socioeconomic status. J Ophthalmol. 2012;2012:298459. A study emphasizing that patient factors such as post-surgical hygiene may play a role in the risk of endophthalmitis.

42. • Zahid S, Musch DC, Niziol LM, Lichter PR. Risk of endophthalmitis and other long-term complications of trabeculectomy in the Collaborative Initial Glaucoma Treatment Study (CIGTS). Am J Ophthalmol. 2013;155:674-80, 80 e1. A prospective study evaluating the risks of endophthalmitis after trabeculectomy.

43. Alwitry A, King AJ. Surveillance of late-onset bleb leak, blebitis and bleb-related endophthalmitis - a UK incidence study. Graefes Arch Clin Exp Ophthalmol. 2012;250:1231-6.

44. Kuang TM, Lin YC, Liu CJ, et al. Early and late endophthalmitis following trabeculectomy in a Chinese population. Eur J Ophthalmol. 2008;18:66-70.

45. Wilkins $\mathrm{M}$, Indar A, Wormald R. Intra-operative mitomycin $\mathrm{C}$ for glaucoma surgery. Cochrane Database Syst Rev. 2005;1:CD002897.

46. Al-Torbak AA, Al-Shahwan S, Al-Jadaan I, et al. Endophthalmitis associated with the Ahmed glaucoma valve implant. Br J Ophthalmol. 2005;89:454-8.

47. Keay L, Gower EW, Cassard SD, et al. Postcataract surgery endophthalmitis in the United States: analysis of the complete 2003 to 2004 Medicare database of cataract surgeries. Ophthalmology. 2012;119:914-22.

48. Freeman EE, Roy-Gagnon MH, Fortin E, et al. Rate of endophthalmitis after cataract surgery in quebec, Canada, 1996-2005. Arch Ophthalmol. 2010;128:230-4.

49. Melo GB, Bispo PJ, Regatieri CV, et al. Incidence of endophthalmitis after cataract surgery (2002-2008) at a Brazilian university-hospital. Arq Bras Oftalmol. 2010;73:505-7. 